\title{
Doença de Cushing Subclínica: Relato de Três Casos e Revisão da Literatura
}

\begin{abstract}
RESUMO
Ao contrário da doença de Cushing subclínica, a síndrome de Cushing subclínica de origem adrenal é uma entidade amplamente conhecida. É definida como uma hiperprodução autônoma de cortisol, de leve intensidade, insuficiente para causar sinais clínicos específicos de hipercortisolismo, porém suficiente para levar a alterações bioquímicas detectáveis no eixo hipotálamo-hipófise-adrenal. Apesar de a doença de Cushing representar a grande maioria dos casos de hipercortisolismo, há poucos relatos na literatura sobre doença de Cushing subclínica. Neste trabalho são descritos três casos de doença de Cushing subclínica, causadas por macroadenomas hipofisários corticotróficos, confirmados por estudo imuno-histoquímico, despertando a atenção dos endocrinologistas clínicos para essa entidade e sugerindo que a investigação laboratorial do hipercortisolismo seja incluída em todos os casos de adenomas hipofisários. (Arq Bras Endocrinol Metab 2007;51/4:625-630)
\end{abstract}

Descritores: Síndrome de Cushing subclínica; Doença de Cushing subclínica; Macroadenoma corticotrófico; Tumor hipofisário; Imuno-histoquímica

\section{ABSTRACT}

\section{Subclinical Cushing's Disease: Presentation of Three Cases and Critical Review.}

Unlike subclinical Cushing's disease, adrenal subclinical Cushing's syndrome is widely recognized. It is defined as an autonomous cortisol hyperproduction of mild intensity not causing specific clinical signs, but detectable biochemically as derangements of the hypothalamic-pituitary-adrenal axis function. Although Cushing's disease accounts for the majority of hypercortisolism states, subclinical Cushing's disease has been rarely reported. Three cases of subclinical Cushing's disease due to pituitary corticotrophic macroadenomas, confirmed by immunohistochemistry, are presented in order to underscore its recognition by clinical endocrinologists and to emphasize a diagnostic evaluation of hypercortisolism in all cases of pituitary adenomas. (Arq Bras Endocrinol Metab 2007;51/4625:-630)

Keywords: Subclinical Cushing's syndrome; Subclinical Cushing's disease; Corticotrophic macroadenoma; Pituitary tumor; Immunohistochemistry

\footnotetext{
Síndrome De Cushing, descrita em 1921 por Harvey Cushing, é o complexo de sintomas e sinais associados à exposição prolongada a quantidades excessivas de glicocorticóides livres plasmáticos (1). Tem como causa principal a administração crônica de glicocorticóides, sendo chamada, nesta situação, de síndrome de Cushing exógena $(1,2)$. A síndrome de Cushing endógena resulta de uma hiperprodução de glicocorticóides pelo córtex da adrenal. Em 15 a $20 \%$ dos casos, essa produção é independente do hormônio adrenocorticotrófico (ACTH), sendo depen-
}

apresentação de caso

\author{
Bárbara C. Carvalho Silva \\ AlFredo M. BARUQUI JÚNIOR \\ VÍCTOR EURÍPEDES BARBOSA \\ Gil Patrus Mundim Pena \\ Marisa Helena de F. Campos \\ ADAUTO VERSIANI RAMOS \\ Eduardo Pimentel Dias
}

Serviço de Endocrinologia da Santa Casa de Belo Horizonte (BCCS), Serviço de

Endocrinologia (AMBJ, VEB, MHFC, AVR \& EPD) e Serviço de Anatomia Patológica (GPMP) do Hospital Felício Rocho, e Instituto Felício Rocho de Pesquisa e Educação Continuada (IFERPEC) (EPD),

Belo Horizonte, MG.

Recebido em 02/09/05

Revisado em 05/09/06

Aceito em 26/02/07 
dente do ACTH nos casos restantes (3). A síndrome de Cushing ACTH-independente é ocasionada por tumores adrenais benignos ou malignos ou por hiperplasia adrenal bilateral. A síndrome de Cushing dependente de ACTH é causada principalmente por tumores hipofisários corticotróficos, produtores de ACTH, definindo a doença de Cushing. Mais raramente, pode ser causada por produção ectópica de ACTH ou por tumores secretores de CRH (3). Os tumores corticotróficos são representados, em sua grande maioria, por microadenomas (90\% dos casos) (2). Os macroadenomas são raros e os carcinomas, extremamente incomuns (4).

Independentemente de sua etiologia, a síndrome de Cushing tem como manifestações clínicas clássicas a obesidade truncal, estrias cutâneas violáceas, giba de búfalo, "face de lua cheia", pletora facial, fraqueza muscular proximal, extremidades finas com hipotrofia muscular, fragilidade capilar, acne, hirsutismo e irregularidade menstrual $(1,2,5)$. Problemas associados, como hipertensão arterial e diabetes mellitus, são comuns (2).

A síndrome de Cushing subclínica é definida como uma hipersecreção autônoma de cortisol, com alterações bioquímicas no eixo hipotálamo-hipófiseadrenal, porém com ausência das manifestações clínicas clássicas da síndrome de Cushing (5). Apesar da ausência dos estigmas clássicos do hipercortisolismo, os pacientes com síndrome de Cushing subclínica têm alta prevalência de obesidade, hipertensão arterial, diabetes e redução da densidade mineral óssea $(5,6)$. A síndrome de Cushing subclínica de origem adrenal vem sendo amplamente descrita e debatida na literatura (5-13). Por outro lado, existem poucos relatos de doença de Cushing subclínica (14-17).

O objetivo deste trabalho é relatar três casos de doença de Cushing subclínica, por macroadenomas corticotróficos, confirmados por imuno-histoquímica, e despertar a atenção dos endocrinologistas clínicos para esta entidade.

\section{MÉTODOS}

\section{Avaliação histológica e imuno-histoquímica}

As amostras foram fixadas em formaliza a $10 \%$ e incluídas em parafina. Cortes histológicos de $5 \mu \mathrm{m}$ foram obtidos e corados por H\&E e submetidos à reação imuno-histoquímica para pesquisa de hormônios hipofisários. Os anticorpos utilizados, diluições e métodos de recuperação antigênica são detalhados na tabela 1 . O sistema de visualização empregado foi o Envision $+{ }^{\circledR}(\mathrm{DAKO})$, com revelação feita pelo método da diamino-benzidina. Controles negativos e positivos foram processados paralelamente à amostra em estudo. Coloração de fundo foi feita com hematoxilina. Em um dos casos, a presença de glândula hipófise não neoplásica no espécime serviu também como controle interno da reação. A reação era considerada positiva quando o citoplasma das células demonstrava nítida coloração amarronzada. A positividade era considerada difusa quando acima de $75 \%$ das células demonstravam reatividade.

\section{Exames laboratoriais}

As determinações laboratoriais foram realizadas por quimioluminescência (cortisol plasmático, cortisol livre urinário, ACTH plasmático, TSH, T4 livre e total), por imunofluorimetria (prolactina, $\mathrm{FSH}$ e $\mathrm{LH}$ ) e por imunorradiometria (IGF-1). Os valores de referência estão listados a seguir:

- Cortisol plasmático basal, medido às 8 horas: 5 a 25 $\mu \mathrm{g} / \mathrm{dl}$

- Cortisol livre urinário: 10 a $90 \mu \mathrm{\mu g} / 24$ horas

- ACTH plasmático: $<46 \mathrm{pcg} / \mathrm{ml}$

- TSH: 0,3 a $5,0 \mu \mathrm{U} / \mathrm{ml}$

- T4 livre: 0,7 a $1,8 \mathrm{ng} / \mathrm{dl}$

- T4 total: 5 a $12 \mu \mathrm{g} / \mathrm{dl}$

- Prolactina: 0,6 a $19,0 \mathrm{ng} / \mathrm{ml}$

- FSH: fase folicular 2,4 a 9,3 U/L; fase ovulatória 3,9 a 13,3 U/L; fase luteínica 0,3 a $8,0 \mathrm{U} / \mathrm{L}$; pós menopausa $>20 \mathrm{U} / \mathrm{L}$

- LH: fase folicular 1,6 a 9,3; fase ovulatória 13,8 a 71,8 $\mathrm{U} / \mathrm{L}$; fase luteínica 0,2 a $12,8 \mathrm{U} / \mathrm{L}$; pós menopausa > 15 $\mathrm{U} / \mathrm{L}$

- IGF-1: mulheres entre 18 e 20 anos: 215 a 794 ng/ml; mulheres entre 50 e 70 anos: 55 a $252 \mathrm{ng} / \mathrm{ml}$.

Os testes dinâmicos de supressão com doses baixas de dexametasona foram realizados administrando-se $1 \mathrm{mg}$ de dexametasona, por via oral às 23 horas, com determinação do cortisol às 8 horas da manhã seguinte ou com a administração, por vai oral, de $0,5 \mathrm{mg}$ de dexametasona de $6 \mathrm{em} 6$ horas por 48 horas, dosando-se o cortisol 6 horas após a última dose. Valores de cortisol plasmático maiores ou iguais a $1,8 \mu \mathrm{g} / \mathrm{dl}$ foram considerados não supressos. O teste de supressão com dose alta de dexametasona foi realizado administrando-se 8 $\mathrm{mg}$ de dexametasona, por via oral, às 23 horas, com a dosagem do cortisol plasmático às 8 horas da manhã seguinte. Supressão do cortisol maior que $50 \%$ em relação ao valor basal foi considerada indicativa de doença de Cushing.

\section{RELATO DE CASOS}

\section{Caso 1}

Paciente L.L.A., 18 anos, sexo feminino, iniciou quadro de irregularidade menstrual aos 15 anos, procurando atendimento médico com ginecologista. Exames solicitados na época: Prolactina $(\mathrm{PRL})=$ $88,5 \mathrm{ng} / \mathrm{ml}$ e $51,9 \mathrm{ng} / \mathrm{ml}$. Tomografia computadorizada de região selar revelou macroadenoma hipofisário (figura 1). 
Tabela 1. Anticorpos, diluição e métodos de recuperação antigênica empregados.

\begin{tabular}{lccccc}
\hline Anticorpo & Clone & Animal & Fornecedor & $\begin{array}{c}\text { Recuperação } \\
\text { antigênica }\end{array}$ & Diluição \\
Anti-ACTH & O2A3 & Camundongo & DAKO & Nenhuma & $1: 800$ \\
Anti-GH & Policlonal & Coelho & DAKO & Nenhuma & $1: 1200$ \\
Anti-PRL & Policlonal & Coelho & DAKO & Nenhuma & $1: 1100$ \\
Anti-FSH & C10 & Camundongo & DAKO & Digestão & $1: 25$ \\
Anti-LH & Policlonal & Coelho & DAKO & Nenhuma & $1: 4000$ \\
\hline Anti-TSH & 0042 & Camundongo & DAKO & Nenhuma & $1: 400$ \\
\hline
\end{tabular}

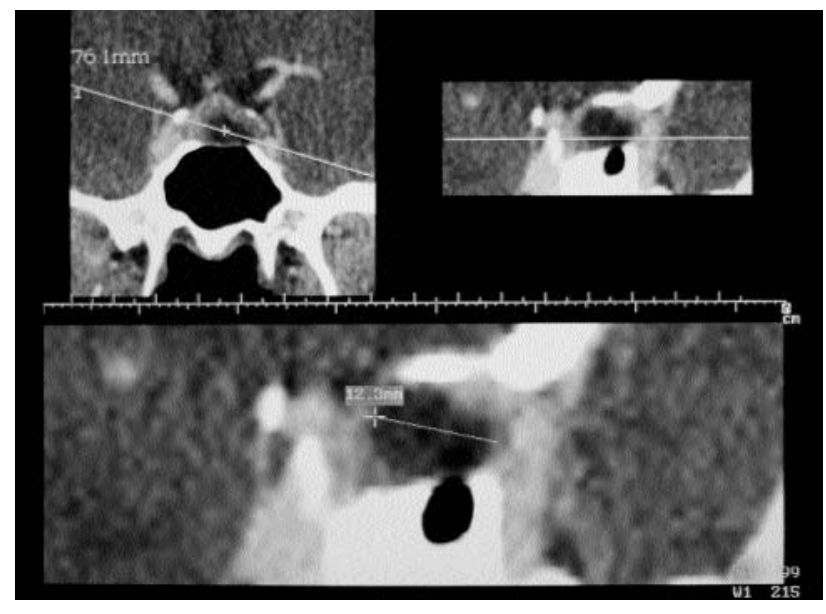

Figura 1. Tomografia computadorizada da sela túrcica no plano coronal, com reconstrução no plano sagital pós-contraste iodado endovenoso, mostrando massa intra-selar com área hipodensa central que pode corresponder a necrose ou degeneração cística.

Feito o diagnóstico de macroprolactinoma, foi iniciada bromocriptina $1,25 \mathrm{mg}$ ao dia com melhora dos sintomas e normalização dos níveis de prolactina $(7,1 \mathrm{ng} / \mathrm{ml}$ em 2003), porém sem alteração do volume tumoral.

Em 2003, procurou o ambulatório do nosso serviço para reavaliação do caso. Negava co-morbidades, estava assintomática e tinha ciclos menstruais regulares, em uso de bromocriptina. Seu índice de massa corporal (IMC) era $19,4 \mathrm{~kg} / \mathrm{m}^{2}$ e não tinha sinais clínicos clássicos de hipercortisolismo. Seu perfil lipídico e glicemia eram normais.

A dose de bromocriptina foi aumentada para 2,5 mg, 2 vezes ao dia, na tentativa de redução tumoral e solicitados exames: $\mathrm{PRL}=1,7 \mathrm{ng} / \mathrm{ml}$, TSH= $0,72 \mu \mathrm{Ui} / \mathrm{ml}, \mathrm{T} 4$ livre $=1,5 \mathrm{ng} / \mathrm{dl}, \mathrm{FSH}=1,43 \mathrm{U} / \mathrm{L}$, $\mathrm{LH}=3,64 \mathrm{U} / \mathrm{L}, \mathrm{IGF}-\mathrm{l}=452 \mathrm{ng} / \mathrm{ml}$, cortisol basal= $18,9 \mu \mathrm{g} /$ dla e ACTH basal= $103 \mathrm{pg} / \mathrm{ml}$. Foi, então, submetida a estudo da dinâmica do eixo hipotálamohipófise-adrenal com os seguintes resultados: cortisol após $1 \mathrm{mg}$ de dexametasona noturna $=10,5 \mu \mathrm{g} / \mathrm{dl}$, cortisol após $0,5 \mathrm{mg}$ de dexametasona de $6 \mathrm{em} 6$ horas por 48 horas $=9,4 \mu \mathrm{g} / \mathrm{dl}$; cortisol após $8 \mathrm{mg}$ de dexametasona noturna $=2 \mu \mathrm{g} / \mathrm{dl}$.

Submetida a cirurgia transesfenoidal em julho de 2003. O resultado anátomo-patológico e imunohistoquímico revelou adenoma corticotrófico, conforme descrição: material constava de vários fragmentos irregulares de tecido, com alterações artefatuais por tracionamento e aspiração, resultando em extravasamento de hemácias, discoesão celular e fragmentação do tecido. A histologia do caso demonstrou pequena área adenomatosa, com padrão de crescimento sólido (figuras $2 \mathrm{~A}$ e $2 \mathrm{~B}$ ), com expressão difusa (acima de $80 \%$ das células) de ACTH (figura 2C), corando todo o citoplasma das células, caracterizando adenoma corticotrófico. A pesquisa dos outros hormônios foi negativa (figuras $2 \mathrm{D}$ e $2 \mathrm{E}$ ). Havia, também, extensas áreas de adeno-hipófise, com grupos celulares distintos demonstrando reação para os diferentes anticorpos pesquisados. Algumas áreas de neuro-hipófise também foram identificadas no material. Em uma dessas áreas, o tecido neural era penetrado por células basofílicas, que, no estudo imuno-histoquímico, reagiram para o anticorpo contra ACTH. Esse fenômeno é conhecido como "invasão dos basófilos" (figuras $2 \mathrm{~F}$ e $2 \mathrm{G}$ ). O significado funcional deste achado não é conhecido, não estando, por si só, associado a quaisquer anormalidades endócrinas.

\section{Caso 2}

Paciente S.M.M., 58 anos, sexo feminino, submetida em 1981 a hipofisectomia para tratamento de provável macroadenoma não secretor com comprometimento do campo visual, sem outras co-morbidades. Permaneceu assintomática até 2003, quando procurou nosso serviço com queixa de hemianopsia bitemporal e cefaléia.

Estava em amenorréia desde os 45 anos, época em que realizou histerectomia por miomatose uterina. Apresentava sobrepeso leve, com IMC de $27,3 \mathrm{~kg} / \mathrm{m}^{2}$, mas as manifestações clínicas clássicas do hipercortisolismo estavam ausentes. Tinha perfil lipídico e gli- 


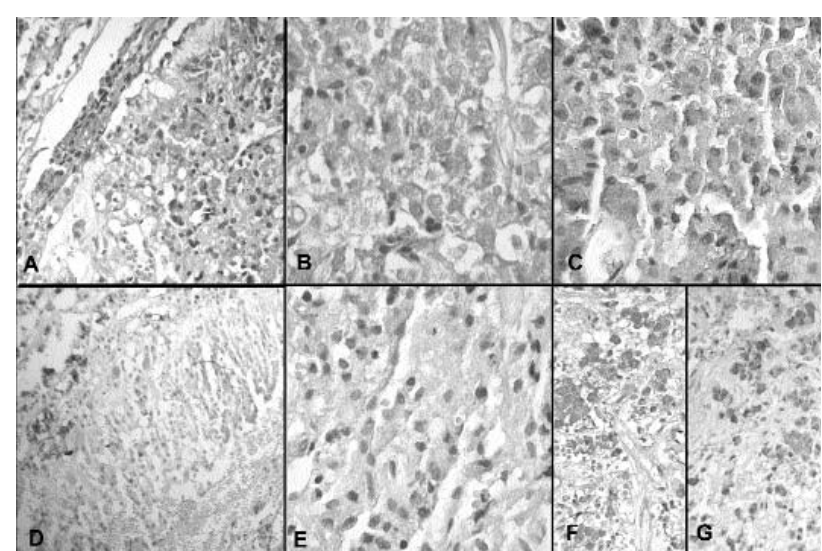

Figura 2. Histologia demonstrando pequena área adenomatosa, com padrão de crescimento sólido (A e B), com expressão difusa (acima de $80 \%$ das células) de ACTH (C), corando todo o citoplasma das células, caracterizando adenoma corticotrófico. Pesquisa dos outros hormônios negativa (D e E). Área de neuro-hipófise, com penetração de células basofílicas no tecido neural, que, no estudo imuno-histoquímico, reagiram para o anticorpo contra $\mathrm{ACTH}$, fenômeno conhecido como "invasão dos basófilos" ( $\mathrm{F}$ e $\mathrm{G}$ ).

cemia normais. A ressonância magnética (RM) da região hipotálamo-hipofisária evidenciava recidiva do tumor com provável invasão do seio cavernoso (figura $3)$. Os exames solicitados revelaram: $\mathrm{FSH}=35 \mathrm{U} / \mathrm{L}$, $\mathrm{PRL}=7,6 \mathrm{ng} / \mathrm{ml}$, IGF- $\mathrm{l}=172 \mathrm{ng} / \mathrm{ml}, \mathrm{TSH}=1,7$ $\mu \mathrm{U} / \mathrm{ml}$ e T4 livre $=1,0 \mathrm{ng} / \mathrm{dl}$. Não foi realizada investigação laboratorial de hipercortisolismo devido à baixa suspeição clínica de doença de Cushing.

Foi submetida a cirurgia transesfenoidal, com hipótese diagnóstica de macroadenoma hipofisário clinicamente não secretor. O resultado anátomopatológico com imuno-histoquímica revelou presença de adenoma corticotrófico, conforme descrição: material recebido para o estudo consistia de fragmentos irregulares de tecido. À histologia, observou-se neoplasia epitelial de padrão adenomatoso, sólido, formada por proliferação de células poligonais, de citoplasma amplo, basofílico (figuras 4A e 4B). O estudo imuno-histoquímico demonstrou expressão difusa (100\% das células) de ACTH nas células do adenoma, caracterizando adenoma corticotrófico (figura 4C). A pesquisa dos demais hormônios foi negativa (figura 4D). Neste material, não foram identificadas áreas de hipófise não neoplásica.

Durante o acompanhamento pós-operatório, paciente teve uma redução ponderal de $10 \mathrm{~kg}$ em um ano, com normalização do IMC.

\section{Caso 3}

Paciente R.C.S.R, 32 anos, sexo feminino, procurou nosso serviço em 1991, com quadro de galactorréia, irregularidade menstrual e cefaléia. Negava hiperten-

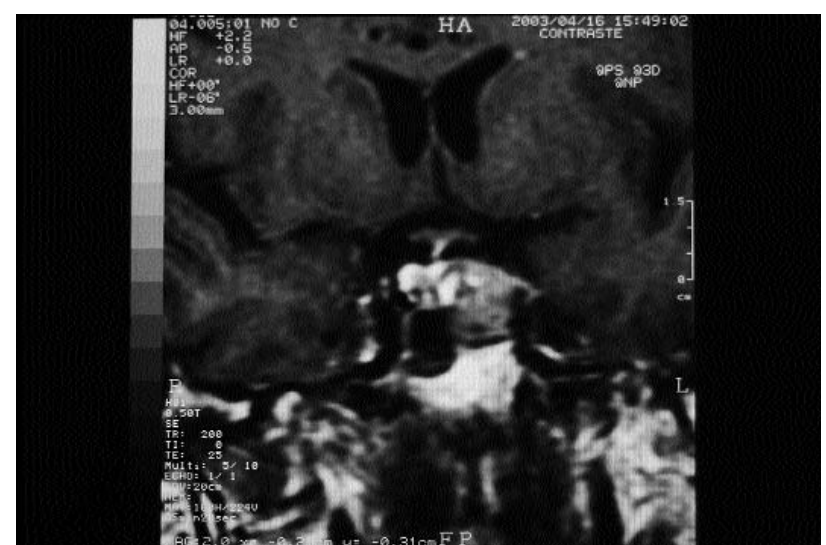

Figura 3. Ressonância magnética de sela túrcica em corte coronal, ponderado em T1 pós-contraste, mostrando massa intra-selar em região lateral esquerda, hipocaptante pelo meio de contraste, estendendo-se para o seio esfenoidal e envolvendo parcialmente o seio cavernoso homolateral.

são ou outras co-morbidades. Tinha IMC de 19,5 $\mathrm{kg} / \mathrm{m}^{2}$ e estavam ausentes os sinais clássicos de hipercortisolismo. Exames realizados na ocasião: PRL $=68$ $\mathrm{ng} / \mathrm{ml}, 74 \mathrm{ng} / \mathrm{ml}$ e $66 \mathrm{ng} / \mathrm{ml}$. RM da região hipotálamo-hipofisária revelou macroadenoma hipofisário (figura 5 ).

Iniciou-se bromocriptina, na dose de $5 \mathrm{mg} / \mathrm{dia}$. Em 1992, novos exames revelaram: $\mathrm{PRL}=1,0 \mathrm{ng} / \mathrm{ml}$, $\mathrm{TSH}=2,4 \mu \mathrm{Ui} / \mathrm{ml}, \mathrm{T} 4$ total $=9,0 \mathrm{ng} / \mathrm{dl}$, cortisol basal $=25 \mu \mathrm{g} / \mathrm{dl}$ e cortisol após $1 \mathrm{mg}$ de dexametasona noturna $=17 \mu \mathrm{g} / \mathrm{dl}$. Repetida RM, que não evidenciou alteração no volume tumoral. Foi, então, encaminhada a cirurgia.

Quatro meses após a cirurgia, paciente estava assintomática e com ciclos menstruais regulares sem uso de bromocriptina. Exames solicitados: $\mathrm{PRL}=8,8$ $\mathrm{ng} / \mathrm{ml}, \mathrm{TSH}=2,6 \mu \mathrm{U} / \mathrm{ml}, \mathrm{T} 4$ total $=7,5 \mathrm{ng} / \mathrm{dl}$, cortisol basal=9,5 $\mu \mathrm{g} / \mathrm{dl}, \mathrm{RM}$ : tecido tumoral aderido ao seio cavernoso direito.

Nova propedêutica para hipercortisolismo demonstrou: cortisol após $1 \mathrm{mg}$ de dexametasona notur$\mathrm{na}=7,3 \mu \mathrm{g} / \mathrm{dl}$, cortisol após $0,5 \mathrm{mg}$ de dexametasona de $6 \mathrm{em} 6$ horas por 48 horas $=8,3 \mu \mathrm{g} / \mathrm{dl}$, cortisol livre urinário $=72,9 \mu \mathrm{c} / 24$ e ACTH plasmático $=51 \mathrm{pg} / \mathrm{ml}$.

$\mathrm{O}$ estudo imuno-histoquímico do tumor foi realizado, em outro serviço, 1 ano após a cirurgia, evidenciando mais de $75 \%$ das células com positividade para ACTH.

\section{DISCUSSÃO}

A síndrome de Cushing subclínica de origem adrenal é uma entidade amplamente conhecida. É definida 


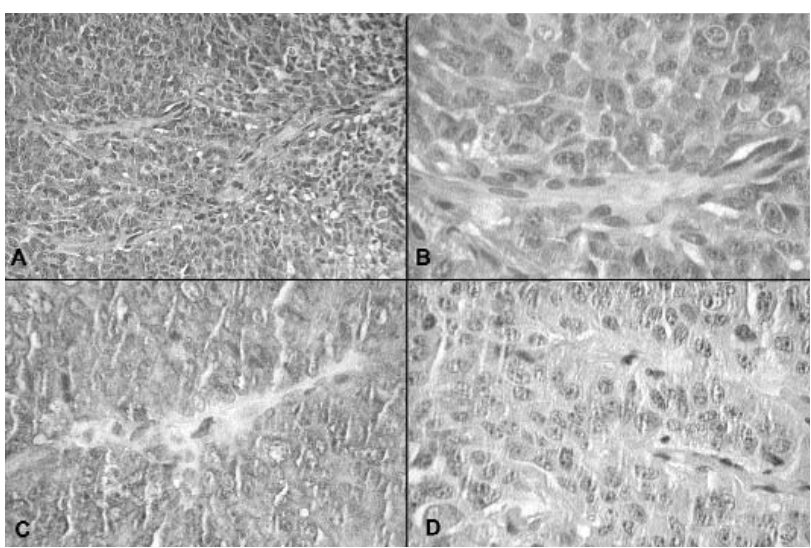

Figura 4. Neoplasia epitelial de padrão adenomatoso, sólido, formada por proliferação de células poligonais, de citoplasma amplo, basofílico (A e B). Estudo imuno-histoquímico demonstrando expressão difusa (100\% das células) de ACTH nas células do adenoma (C). Pesquisa dos demais hormônios negativa (D).

como uma hiperprodução autônoma de cortisol, de leve intensidade, insuficiente para causar sinais clínicos específicos de hipercortisolismo, porém suficiente para levar a alterações bioquímicas detectáveis no eixo hipotálamo-hipófise-adrenal (5). Tem uma prevalência estimada de 79 casos por 100.000 pessoas, sendo muito mais comum que a síndrome de Cushing clássica $(6,12)$.

Por outro lado, apesar de a doença de Cushing ser a causa mais comum de hipercortisolismo (3), há poucos relatos na literatura sobre doença de Cushing subclínica (14-17). Takao e col. (14) sugerem os seguintes critérios para o diagnóstico de doença de Cushing subclínica: 1) ausência de aparência $\mathrm{Cu}^{-}$ shingóide, 2) ACTH e cortisol basal plasmáticos normais, 3) perda do ritmo circadiano normal de cortisol e ACTH, 4) resposta exagerada do ACTH plasmático à desmopressina e ao $\mathrm{CRH}$ e 5) supressão incompleta do cortisol com baixas doses de dexametasona.

Os autores descrevem três casos de doença de Cushing, em pacientes sem estigmas de hipercortisolismo, com imuno-histoquímica diagnóstica de adenoma corticotrófico e com cortisol plasmático não supressível com baixas doses de dexametasona em dois deles (casos 1 e 3). Diferentemente dos casos relatados na literatura que tratam de doença de Cushing subclínica associada a microadenomas (14-17), nossa casuística é exclusivamente de macroadenomas.

A progressão da síndrome de Cushing subclínica para a forma manifesta da síndrome de Cushing é alvo de debate na literatura. Alguns autores consideram o risco dessa progressão muito baixo ou quase nulo $(7,9,10)$, mas Barzon e cols. (12) encontraram

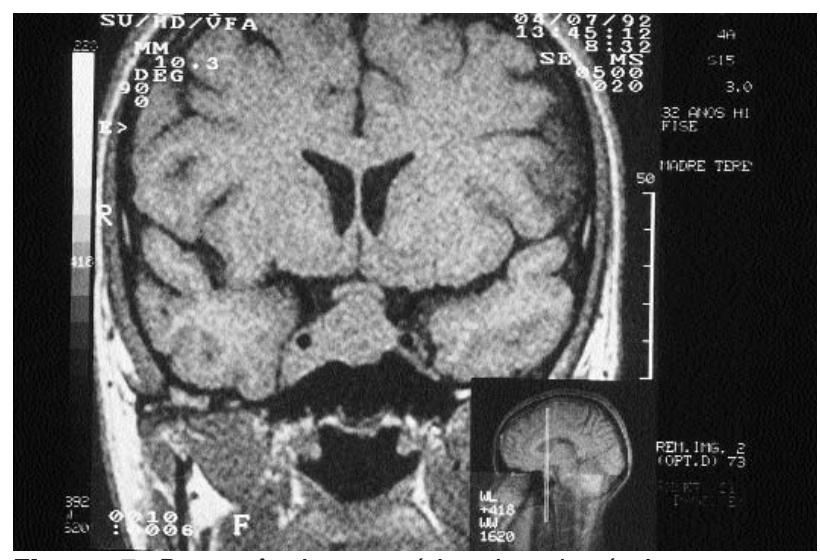

Figura 5. Ressonância magnética de sela túrcica em corte coronal, ponderado em T1, mostrando volumosa massa intra e supra-selar condicionando infra-desnivelamento do assoaIho selar e envolvendo a artéria carótida interna direita.

um risco cumulativo de $12,5 \%$ após 1 ano de diagnóstico de síndrome de Cushing subclínica em pacientes com incidentaloma adrenal. Em relação à doença de Cushing subclínica, essa evolução é apenas hipotética, uma vez que não há relato de casos dessa entidade sem tratamento e com acompanhamento a longo prazo.

Sabe-se que a síndrome de Cushing subclínica de origem adrenal está associada à hipertensão arterial, diabetes tipo 2 , intolerância à glicose, obesidade visceral, perfil lipídico desfavorável e redução da massa óssea $(5,7,9)$. Esses achados sugerem que os pacientes com síndrome de Cushing subclínica têm um risco cardiovascular aumentado, de forma semelhante ao que ocorre entre os pacientes com síndrome de $\mathrm{Cu}-$ shing manifesta, e que o tratamento cirúrgico reduziria este risco (5).

Nagai e col. (15) relataram um caso de hipertensão arterial maligna e diabetes mellitus tipo 2, associados à doença de Cushing subclínica. Após tratamento cirúrgico, houve melhora dos níveis pressóricos e do controle glicêmico.

Recentemente, Catargi e col. (17) identificaram 4 pacientes com síndrome de Cushing em uma série de 200 pacientes internados com diagnóstico de diabetes mellitus tipo 2 mal controlados. Desses, 3 tinham doença de Cushing por microadenomas hipofisários, comprovada por exame anátomo-patológico em 2 casos e por cateterismo bilateral do seio petroso inferior em um dos pacientes que não pôde ser operado. Esses pacientes foram tratados cirurgicamente, com radioterapia e cetoconazol, isolados ou em conjunto. Após 6 meses do tratamento, foi observada uma redução média do peso corporal em $5,5 \%$ e da Alc em 2,5\%. 
Apesar da ausência de evidências bem sedimentadas que comprovem um aumento do risco cardiovascular em pacientes portadores de doença de Cushing subclínica, existem alguns indícios que corroboram essa hipótese $(15,17)$. Paralelamente, extrapolando os achados de risco cardiovascular aumentado em pacientes com incidentaloma adrenal (5) para os pacientes com doença de Cushing subclínica, confirmaríamos a necessidade de um tratamento eficaz nesses casos, de preferência cirúrgico, no sentido de corrigir o hipercortisolismo.

Conclui-se que a doença de Cushing subclínica pode ocorrer em pacientes com micro ou macroadenomas hipofisários, e é provável que seja mais comum que o relatado na literatura atual, possivelmente em função do seu subdiagnóstico. A importância de sua identificação se faz pela necessidade da instituição de um tratamento efetivo, devido a sua maior associação com outras co-morbidades, como hipertensão arterial, diabetes tipo 2, osteoporose e doenças cardiovasculares, e pela possibilidade de progressão para doença de Cushing manifesta. A fim de se evitar o subdiagnóstico da doença de Cushing subclínica, sugere-se como propedêutica mínima de qualquer adenoma hipofisário, inclusive os achados incidentalmente, não apenas a dosagem de prolactina, como proposto por alguns autores $(18,19)$, mas também a determinação de cortisol após supressão com 1 mg de dexametasona noturna e a investigação do ritmo circadiano do cortisol, com determinação do cortisol basal e à meia-noite.

\section{AGRADECIMENTOS}

Os autores agradecem à Dra. Juliana Beaudette Drummond e ao Dr. Cristiano Alvarenga Rocha pela assistência técnica prestada, e ao Dr Júlio Abucham pela realização da imuno-histoquímica do caso 3 .

\section{REFERÊNCIAS}

1. Steward PM. The Adrenal Cortex. In: Larsen R, Kronenberg HM, Melmed S, Polonsky KS, eds. Williams Textbook of Endocrinology. $10^{\text {th }}$ ed. Philadelphia: Saunders, 2003. pp. 491-551.

2. Vilar $L$, Coelho CE. Diagnóstico e diagnóstico diferencial da Síndrome de Cushing. In: Vilar L, ed. Endocrinologia clínica. 3a ed. Rio de Janeiro: Guanabara Koogan, 2006. pp. $397-$ 417.
3. Arnaldi G, Angeli A, Atkinson AB, Bertagna X, Cavagnini F, Chrousos GP, et al. Diagnosis and complications of Cushing's syndrome: a consensus statement. J Clin Endocrinol Metab 2003;88:5593-602.

4. Rubatino Jr AC, Pereira RF, Benchimol I, Laun IC. Doença de Cushing por macroadenoma hipofisário. Arq Bras Endocrinol Metab 2004;48(6):909-12.

5. Tauchmanovà L, Rossi R, Biondi B, Pulcrano M, Nuzzo V, Palmieri EA, et al. Patients with subclinical Cushing's syndrome due to adrenal adenoma have increased cardiovascular risk. J Clin Endocrinol Metab 2002;87:4872-8.

6. Reincke M. Subclinical Cushing's syndrome. Endocrinol Metab Clin North Am 2000;29(1):43-56.

7. Terzolo M, Pia A, Ali' A, Osella G, Reimondo G, Bovio S, et al. Adrenal Incidentaloma: a new cause of the metabolic syndrome? J Clin Endocrinol Metab 2002;87:998-2003.

8. Terzolo M, Reimondo G, Bovio S, Angeli A. Subclinical Cushing's syndrome. Pituitary 2004;7(4):217-23.

9. Rossi R, Tauchmanovà L, Luciano A, Di Martino M, Battista C, Del Viscovo $L$, et al. Subclinical Cushing's syndrome in patients with adrenal incidentaloma: clinical and biochemical features. J Clin Endocrinol Metab 2000;85:1440-8.

10. Terzolo M, Osella G, Ali A, Borreta G, Cesario F, Pacotti P, et al. Subclinical Cushing's syndrome in adrenal incidentaloma. Clin Edocrinol (Oxf) 1998;48(1):89-97.

11. Bovio S, Reimondo G, Daffara F, Allasino B, Angeli A, Terzolo M. Subclinical Cushing's syndrome in adrenal incidentalomas. Recenti Prog Med 2006;97:6-15.

12. Barzon L, Fallo F, Sonino N, Boscaro M. Development of overt Cushing's syndrome in patients with adrenal incidentaloma. Eur J Endocrinol 2002;146:61-6.

13. Chiodini I, Torlontano M, Scilliatani A, Arosio M, Bacci S, Di Lembo $\mathrm{S}$, et al. Association of subclinical hypercortisolism with type 2 diabetes mellitus: a case control study in hospitalized patients. Eur J Endocrinol 2005;153:837-44.

14. Takao T, Mimoto T, Yanamoto M, Hashimoto K. Preclinical Cushing disease. Arch Intern Med 2001;161:892-3.

15. Nagai T, Imamura M, Misumi S, Mori M. Subclinical Cushing's disease accompanied by malignant hypertension and diabetes mellitus. Intern Med 2002;41:566-70.

16. Kageyama K, Moriyama T, Sakihara S, Kawashima S. A case of preclinical Cushing's disease, accompanied with thyroid papillary carcinoma and adrenal incidentaloma. Endocr $\mathbf{J}$ 2003;50:325-31.

17. Catagari B, Rigalleau V, Poussin A, Ronci-Chaix N, Bex V, Vergnot $V$, et al. Occult Cushing's syndrome in type-2 diabetes. J Clin Endocrinol Metab 2003;88:5808-13.

18. King Jr JT, Justice AC, Aron DC. Management of incidental pituitary microadenomas: a cost-effectiveness analysis. $\mathbf{J}$ Clin Endocrinol Metab 1997;82:3625-32.

19. Aron DC. Pituitary incidentalomas. Clin Endocrinol Update 2005;333-8.

Endereço para correspondência:

Víctor Euripedes Barbosa

Rua Uberaba 370, sala 704

30180-080 Belo Horizonte, MG

E-mail: carbar@uai.com.br 